\title{
Editorial to the Topical Collection: Role of Sample Return in Addressing Major Questions in Planetary Sciences
}

\author{
Mahesh Anand ${ }^{1,2}$. Sara Russell ${ }^{2}$. Yangting Lin $^{3}$. \\ Meenakshi Wadhwa ${ }^{4}$ Kuljeet Kaur Marhas ${ }^{5}$. \\ Shogo Tachibana ${ }^{6,7}$
}

Published online: 27 July 2020

(c) Springer Nature B.V. 2020

Major advances in our understanding of the origin and evolution of the inner Solar System have been made through analyses of samples from the Moon returned by the Apollo and Luna missions in 1960s and 1970s. More recently, the Stardust and Genesis missions sampled cometary grains and solar wind particles, respectively, revealing their elemental and isotopic compositions. Similarly, samples returned by the Hayabusa mission from the near-Earth asteroid 25143 Itokawa enabled insights into regolith evolution on minor bodies in the Solar System and provided the first direct link between meteorites and asteroids. As we write this editorial, Hayabusa2 is on its return journey to Earth after having sampled asteroid 162173 Ryugu while OSIRIS-REx is preparing to collect samples from asteroid 101955 Bennu and Chang'E-5 mission is being readied for a robotic sample return from the Moon after a hiatus lasting over 4 decades. Indeed, NASA's recently announced ARTEMIS program with the aim of landing the first woman and the next man on the Moon by 2024

Role of Sample Return in Addressing Major Questions in Planetary Sciences

Edited by Mahesh Anand, Sara Russell, Yangting Lin, Meenakshi Wadhwa, Kuljeet Kaur Marhas and Shogo Tachibana

$凶$ M. Anand

Mahesh.Anand@open.ac.uk

1 Planetary and Space Sciences, School of Physical Sciences, Walton Hall, The Open University, Milton Keynes, MK7 6AA, UK

2 Department of Earth Sciences, The Natural History Museum, Cromwell Road, London SW7 5BD, UK

3 Key Laboratory of Earth and Planetary Physics, Institute of Geology and Geophysics, Chinese Academy of Sciences, Beijing, China

4 School of Earth and Space Exploration, Arizona State University, Tempe, AZ 85287, USA

5 Planetary Sciences Division, Physical Research Laboratory, Ahmedabad, Gujarat 380009, India

6 UTokyo Organization for Planetary and Space Science, The University of Tokyo, Tokyo, Japan

7 Institute of Space and Astronautical Science, Japan Aerospace Exploration Agency, Sagamihara, Japan 
marks the next major phase of human exploration of the Solar System which will undoubtedly lead to the return of new samples from the Moon and is also expected to accelerate efforts towards the first Mars Sample Return (MSR) mission before the end of this decade.

Although lacking a parent body context, meteorites represent another type of "returned" samples that are either "finds" (collected from various locations on Earth, either through dedicated search efforts or through serendipity) or are "falls" (observed to arrive on Earth, almost always without any forewarning but often in a spectacular fashion!). Meteorite research has been invaluable in addressing a number of scientific topics related to Solar System formation and evolution, ranging from nucleosynthetic processes that contributed to materials forming the solar nebula through studies of pre-solar grains to processes (such as fluid-rock interactions and impacts) occurring on rocky parent bodies over the $4.5 \mathrm{Ga}$ history of the Solar System. Indeed, some of the most exciting findings and developments of new concepts have been brought about as a result of painstaking research on meteorites in Earth-based laboratories.

Perhaps one of the most prolific outcomes from recent technological advances has been in our ability to send spacecraft missions (orbiters and landers) for remote and/or in-situ investigations of almost all major bodies in the Solar System, including the first audacious landings on a comet (67P/Churyumov-Gerasimenko) and on Saturn's moon, Titan. Consequently, there now exist global remote sensing datasets at various scales and resolutions for a number of planetary bodies that weren't available previously, and these have already played (and will continue to play) a vital role in the selection of targets for sample return missions.

Notwithstanding the existence of a wealth of remote sensing and cosmochemical data on various Solar System objects (e.g., Moon, Mars, asteroids), significant gaps remain in our understanding of the origin and evolution of the Solar System. These outstanding science questions can only be addressed through high-precision and highly accurate measurements of returned samples for their mineralogical, chemical, and isotopic compositions from the macroscopic to the atomic scales using the most advanced laboratory instruments on Earth. Furthermore, as amply demonstrated by the Apollo and Luna missions, sample return provides us the critical "ground truth" about the visited body, verifying and validating conclusions that can be drawn by remote sensing (both Earth-based and by spacecraft) and via landed instruments on other bodies (perhaps the best example being the application of a calibrated lunar crater chronology to the surfaces of other inner Solar System bodies, most notably Mars). Returned samples further enhance the scientific value of extra-terrestrial materials such as meteorites and cosmic dust by providing additional clues about their parent bodies. Arguably, the biggest benefit arising from sample return missions is the fact that the returned samples can be preserved for decades and used by future generations of explorers to answer questions that haven't been framed yet using laboratory instruments that haven't even been imagined - fitting the metaphor "the gift that keeps on giving".

In this context, a workshop was organised in February (5-9) 2018 at the International Space Science Institute (ISSI), Bern, Switzerland to review our current knowledge and understanding of Solar System formation and evolution and identify major outstanding science questions that remain to be answered through future sample return missions. This joint ISSI-Europlanet workshop was attended by 39 scientists, representing a broad cross section of the international planetary science community. The career stages of attendees ranged from senior researchers to early stage researchers, including PhD students. The Workshop was structured around three main themes: (i) Initial conditions and workings of the Solar System, (ii) Planetary habitats, and (iii) Sample curation. The presentations and associated discussions in these themes were focused on: (1) reviewing major outstanding science questions in the planetary sciences that can only be answered via sample return missions, (2) 
identifying specific target body(ies) for answering specific science question(s), and (3) evaluating and considering sample curation facilities relevant for such sample return missions. An outcome from the discussions and debate held at the workshop has been the publication of 14 peer-reviewed papers in Space Science Reviews and subsequently regrouping of these papers under the Topical Collection "Role of Sample Return in Addressing Major Questions in Planetary Sciences" and co-published as Volume 74 of the Space Sciences Series of ISSI (SSSI) by Springer Verlag.

The 2020s may usher in a new golden era of sample return. Although many such missions are spearheaded by individual space agencies, almost all of them have contributions from one or more international partners. This is an extremely positive development as it signifies that in the 21 st century space exploration is envisaged as a common goal for humanity which can only be achieved through global cooperation. As scientific research on the Apollo and Luna samples continues to demonstrate, collaboration throughout the international science community is indispensable in order to make progress in science and the society.

The main purpose here is to present a collection of scientific papers reviewing the stateof-the-art in our understanding of the origin and evolution of the Solar System and then highlighting major gaps that can only be addressed by returning and analysing samples in Earth-based laboratories. The depth and breadth of each article are likely to provide a firm basis for formulating strategies for future sample return missions by various space agencies.

Mezger et al. review our present understanding of the components that accreted to form the Earth - arguably the most extensively studied planetary body in the Solar System, especially through laboratory analysis of samples. They conclude that it is currently not possible to fully define and quantify the different chemical and isotopic materials that formed the Earth, because a major component seems missing in the extant collections of extraterrestrial samples. They identify Mercury and Venus as planets of great importance and interest in this respect.

Ireland et al. provide a thorough review of the remarkable utility of oxygen isotopic composition of extraterrestrial samples in elucidating some of the most fundamental planetary processes in the Solar System. Central to their argument is the observation that within the chondrite parent bodies, exchange between ${ }^{16} \mathrm{O}$-poor fluids and relatively ${ }^{16} \mathrm{O}$-rich solids created isotopic mixing lines. This model makes specific predictions for isotopic compositions of silicates and water ice throughout the Solar System. Although analysis of returned samples is the ultimate goal, these authors discuss deployment of a promising instrument for three oxygen isotope analysis using a landed mission which could be a precursor to a more challenging and ambitious sample return mission from the visited body.

Vander Kaaden et al. make a compelling case for sample return from Mercury - one of the least studied objects in the inner Solar System. According to these authors, a returned sample from the surface of Mercury would allow for transformative Solar System science, including new insights into space weathering processes on airless bodies and the geochemical behaviour of elements under extreme conditions.

Venus is sometimes referred to as the Earth's "sister planet." Greenwood and Anand argue that the single measurement $\left(\Delta^{17} \mathrm{O}\right)$ of a solid sample of Venus would have first order implications for our understanding of how large terrestrial planets such as our own Earth are built. They review the surface geology of Venus with the aim of identifying potential terrains that could be targeted by a robotic sample return mission. They make a strong case for a Venus sample return using a "Grab and Go" strategy, elements of which have already been studied as part of various mission concept studies by NASA and ESA - so it may not be as outrageous as it may sound, initially!

As the only planetary body other than the Earth where humans have conducted field work and collected samples for laboratory analyses, the Moon has become a reference point for 
understanding planetary processes, serving multiple purposes (e.g., as a cornerstone, testbed, witness plate) for advancing our understanding of Solar System science. Tartèse et al. undertake a heroic task of distilling the vast amount of scientific information that has been derived from investigations of samples returned from the Moon over the past five decades. However, many new and fundamental questions remain which are difficult to address with currently available samples. For example, the abundance, distribution, and source(s) of volatiles on the Moon can only be fully addressed by sampling areas of the Moon not visited previously.

As the speculations grow that astrobiological signatures of the early Earth might be preserved in the lunar regolith, a similar possibility exists for the martian system in which the regolith on its largest moon, Phobos, could have preserved signatures of any life that might have existed on early Mars. Usui et al. describe the scientific objectives of a Japan Aerospace Exploration Agency (JAXA)-led Phobos sample return mission (MMX: Martian Moons eXploration), which aims to retrieve and return $>10 \mathrm{~g}$ of Phobos regolith back to Earth in 2029.

The geological evidence suggesting that Mars was once a habitable planet, and could still harbour liquid water in its sub-surface, raises the tantalising possibility of answering the age-old question that humanity has faced, i.e., "is there life elsewhere in the universe?" As a result, Mars is currently the most intensively explored object in the Solar System with multiple ongoing and planned orbiter and landed missions. The next logical step in martian exploration is a Mars sample return (MSR) mission. Grady provides a review of the science rationale on behalf of the international Mars exploration community that should see the first mission in the MSR campaign, Mars 2020, landing at Jezero Crater in early 2021; samples are anticipated to return to Earth sometime after 2032.

Cockell et al. discuss the microbial ecology of volcanic rocks and hydrothermal systems on the Earth, relevant to developing a sampling strategy for martian samples of astrobiological importance. They suggest that a microbial ecology-driven strategy for investigating the habitability and presence of life on Mars is likely to yield the most promising sample set of the greatest use to the largest number of astrobiologists and planetary scientists.

Kleine et al. review one of the most vigorously debated recent topics in cosmochemistry wherein the isotopic dichotomy between non-carbonaceous (NC) and carbonaceous (CC) meteorites is thought to represent parent bodies from two genetically distinct reservoirs which were isolated within a few million years of the formation of the Solar System. The growth and/or migration of Jupiter subsequently resulted in inward scattering of CC bodies, which accounts for the co-occurrence of $\mathrm{NC}$ and $\mathrm{CC}$ bodies in the present-day asteroid belt. This is also reflected in the composition of the Earth's primitive mantle which has a mixed $\mathrm{NC}-\mathrm{CC}$ composition, indicating that Earth accreted CC bodies during the final stages of its growth, perhaps through the Moon-forming giant impactor.

Burbine and Greenwood make persuasive arguments for returning samples from a mainbelt asteroid. They argue that returning samples from the main belt has the potential to test different planet migration models and the related geochemical and isotopic concept of a bimodal Solar System represented by the NC-CC dichotomy. They advocate a dedicated sample return mission to the dwarf planet (1) Ceres (recently visited by Dawn mission) as the best means of further exploring inherent Solar System variations and conclude that no other potential main-belt target is capable of providing as much insight into the early Solar System as Ceres.

Lin et al. adopt a different approach and make a strong case for developing concepts for sample return missions from asteroids that could reveal the first $10 \mathrm{Ma}$ history of the Solar System, including the initial conditions, nebular processes, and the accretion of planetesimals. In order to "see through" secondary processes that took place in planetesimals, 
such as thermal metamorphism, aqueous alteration, melting and differentiation, they favour targeting primordial objects such as enstatite chondrite-like or L-type Near-Earth asteroids.

Martins et al. review the similarities and differences for organic matter among various Solar System bodies such as comets, asteroids, meteorites, and dust particles in the context of detecting the most pristine organic material, either by on-site analysis or sample return missions. They also discuss the targets of potential future sample return missions, as well as the contamination controls that should be in place in order to successfully study pristine organic matter.

While staying with the topic of organic matter in the Solar System, Chan et al. review the state-of-the-art analytical protocols that are in use for analysing organics in extraterrestrial samples. One of the most challenging aspects of any sample return mission is the requirement to avoid or minimise terrestrial organic contamination. Despite various efforts in the design and execution of contamination control, it appears impossible to completely eliminate sources of organic contamination. Nevertheless, there have been some successes and lessons learned with regard to the identification of indigenous organic matter in the returned samples versus terrestrial contamination.

Sample curation is an indispensable element of any sample return mission plan. A wellplanned sample curation strategy plays a vital role in ensuring the lasting scientific legacy of returned samples for several generations, as exemplified by the NASA's Astromaterials Research and Exploration Science (ARES) program. McCubbin et al. provide a detailed account of astromaterials acquisition and curation practices, especially in the context of advanced curation of astrobiologically sensitive samples that might be returned by future missions. These efforts require research and development of new innovative technologies and techniques for sample collection, handling, characterization, analysis, and curation of astromaterials. The primary result of advanced curation research is to both reduce and quantify contamination of astromaterials and preserve the scientific integrity of all samples from mission inception to secure delivery of samples to Earth-based laboratories for in-depth scientific analysis.

Publisher's Note Springer Nature remains neutral with regard to jurisdictional claims in published maps and institutional affiliations. 